\title{
Fabrication and testing of polymeric membranes for energy efficient separation of Carbon Dioxide from flue gas.
}

Sara Elhoshee, Amna Alabdulla , Fatima Taqi , Dr.Mohamed Hassan, Azza Mohamed Saad

\section{Introduction}

The major anthropogenic Green House Gas in the last few years according to the recent studies is carbon dioxide gas , accounting for $77 \%$ of the human contribution to the greenhouse effect. As illustrated in the pie chart below, the main emissions of $\mathrm{CO} 2$ come from the combustion of fossil fuels.

Polymeric membranes can be synthesized and used for efficient separation of carbon dioxide from light gases. This will lead to a significant reduction in carbon dioxide emission causing green house effect. Furthermore, the separated carbon dioxide can be used in different industries and applications. This technology of polymeric membrane separation will be a new possible strategy to reduce the harmful effects of carbon dioxide emission from fuel combustion. This reduction in carbon dioxide concentration in the atmosphere will definitely benefit the environment to face the global warming
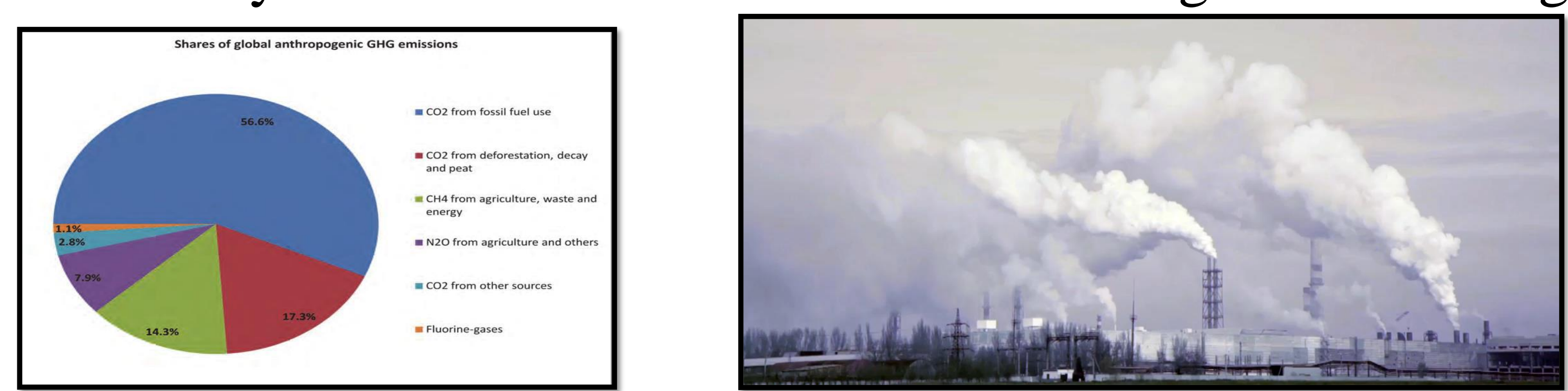

Figure 1: Pie chart showing the shares of anthropogenic GHG emissions.

\section{Research Objectives:}

\footnotetext{
1. Fabrication of polymeric membranes for Carbon dioxide separation.

2. Testing the properties of the prepared membranes.

3. Separation of $\mathrm{CO} 2$ from Nitrogen (flue gas).

4. Demonstrating the potential of this technology to Qatar.
}

\section{Experimental setup}

\section{Materials:}

-PEG diene

Triethyleneglycoldivinylether(TEGDVE)

-PEG di-thiolcrosslinker

2,2-(Ethylenedioxy)Diethanethiol(EDDT)

-Tri-thiolcrosslinker

Trimethylolpropanetri(3-mercaptopropionate) (3T)

-Catalyst

2,2-Dimethoxy-2-Phenylacetophenone(DMPA)

\section{Method:}

\section{Experimental}

- Ultraviolet (UV) curing technique is used to develop advanced polymeric membranes.

- Stability testing is done using Thermogravitationalanalysis (TGA).

-Tensile strength is measured by mechanical tensile experiments.

- Selectivity and permeability are tested using Micro Gas

Chromatography.

\section{Results and Discussion}

- Fabrication of polymeric membranes is simple and economic.

-The fabricated membranes showed good results in thermal stability, tensile strength and performance in separation.

-The separation of carbon dioxide from light gases using polymeric

membranes is energy-efficient.

- The separated carbon dioxide can be used in many other industries and this separation will lead to reduction in global warming.

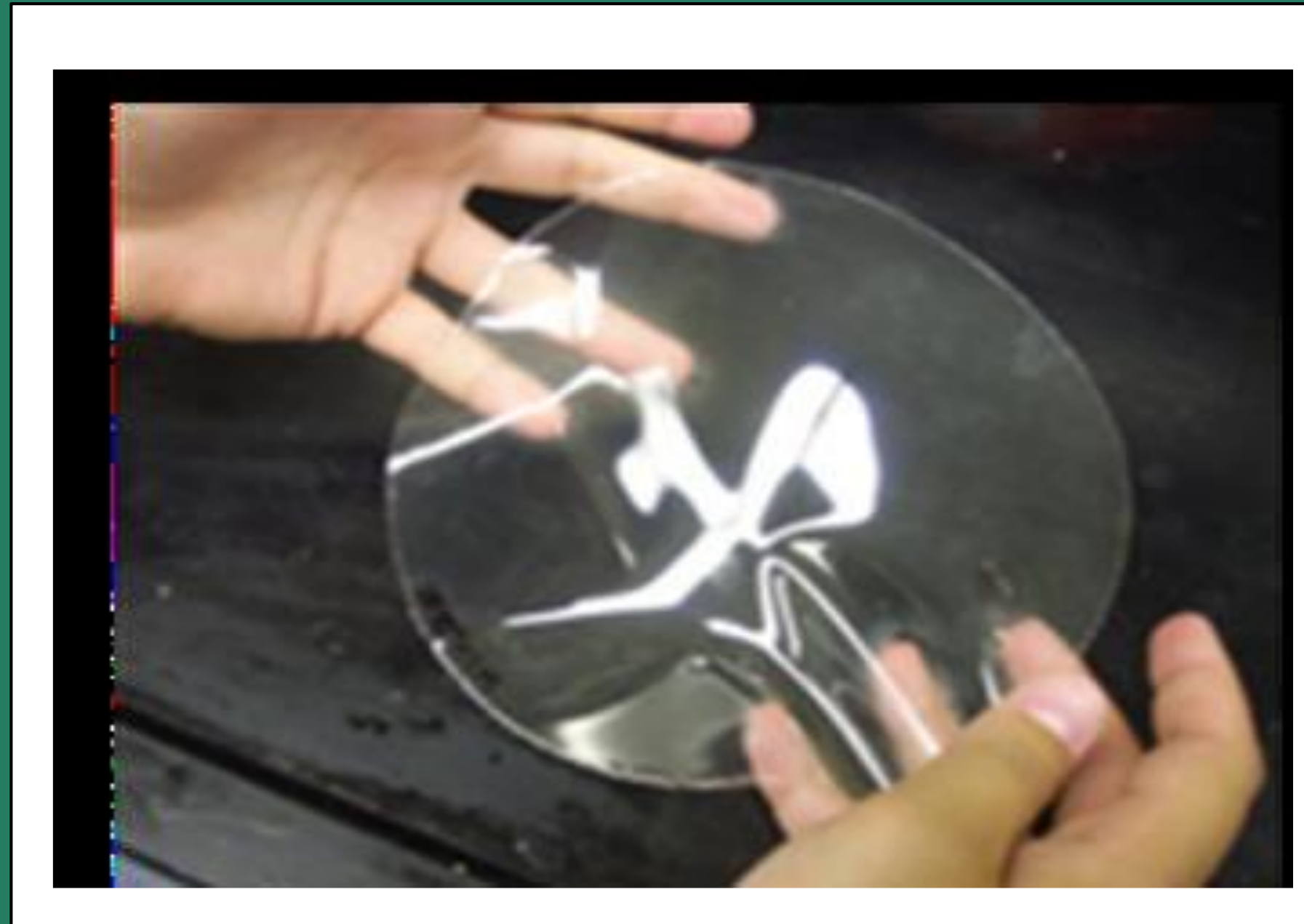

Figure 2: Sample for a polymeric membrane to be tested for carbon dioxide separation.

\begin{tabular}{|l|c|c|c|}
\hline Samples & $\begin{array}{c}\text { Young's } \\
\text { Modulus } \\
(\mathrm{MPa})\end{array}$ & $\begin{array}{c}\text { Elongation at } \\
\text { break } \\
(\%)\end{array}$ & $\begin{array}{c}\text { Tensile } \\
\text { Strength } \\
\text { (MPa) }\end{array}$ \\
\hline O EDDT & 7.32 & 16.95 & 1.11 \\
60 EDDT & 4.73 & 25.71 & 1.13 \\
90 EDDT & 1.89 & 98.76 & 1.27 \\
\hline
\end{tabular}

Figure 4: A table showing the results for mechanical tensile strength testing.

\section{Summary and Future Directions}

Our Research focus on one of the technologies for post combustion capture of carbon dioxide gas from flue gases which is membrane separation In this research, We fabricated four samples of polymeric membranes with different proportions of the components and then tested them for thermal stability, tensile strength selectivity and permeability.

The membrane can be modified by trying different mixtures of the forming polymers with different percentages.

The separated Carbon dioxide gas can be used in different applications like fire extinguishers, carbonated beverages or cooling systems.

Finding more applications for the use of the separated carbon dioxide gas will benefit the environment and will make this project more successful.

The same techniques could be used to fabricate membranes for purifying the methane gas.

Further studies must be done to ensure the effectiveness of these membranes when used in the industry.

\section{Acknowledgements}

This research is supported, and under the supervision of Al-Bairaq project, Center for Advanced Materials (CAM), Qatar University. This project was made possible by NPRP Grant \#[NPRP 10-0126170257] from the Qatar National Research Fund (member of Qatar Foundation)

We thank Dr. Noora Al-Thani, Director of Young Scientists Center (YSC), Qatar University for providing the participated high school students this great opportunity to learn the basics of scientific research.

We are immensely grateful to Dr. Mohamed Hassan, for all the insight, expertise and support he has provided in order to execute this research.

\section{References}

Baker,R.W.FutureDirectionsofMembraneGasSeparationTechnology.Industrial\&EngineeringChemistryResearch2002 B 1,1393-1411. esearch2008,47,2109-2121.

3.Alivisatos,P.:Buchanan,M.Basicresearchneedsforcarboncapture:beyond2020;USDOEOfficeofScience,UnitedStates:

4.Koros,W.J.;Fleming,G.K.Membrane-basedgasseparation.JournalofMembraneScience 1993,83,1-80
. 5.Yampolskii,Y.PolymericGasSeparationMembranes.Macromolecules2012,45,3298-3311 .Bernardo,P.;Drioli,E.;Golemme,G.MembraneGasSeparation:AReview/StateoftheArt.Industrial\&EngineeringChemist

.Brunetti,A.;Scura,F.;Barbieri,G.;Drioli,E.MembranetechnologiesforCO2separation.JournalofMembraneScience2010 